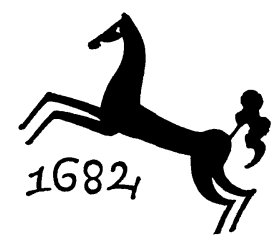

Germanistische AbHandlungen<smiles>C1CCCC1</smiles>

Die deutsche Minneallegorie 
WALTER BLANK

\title{
Die deutsche Minneallegorie
}

\author{
GESTALTUNG UND FUNKTION \\ EINER SPÄTMITTELALTERLICHEN DICHTUNGSFORM
}

J. B. METZLERSCHE

VERLAGSBUCHHANDLUNG

STUTTGART 
Germanistische AbHandLungen 34

Als Habilitationsschrift auf Empfehlung der Philosophischen Fakultät III der Universität Freiburg i. Br.

(C) Springer-Verlag GmbH Deutschland 1970

Ursprünglich erschienen bei J. B. Metzlersche Verlagsbuchhandlung

und Carl Ernst Poeschel Verlag GmbH in Stuttgart 1970

ISBN 978-3-476-99738-8 ISBN 978-3-476-99737-1 (eBook)

DOI 10.1007/978-3-476-99737-1 
MEINER FRAU 


\section{VORWORT}

Diese Arbeit ist mancher wissenschaftlichen Anregung wie auch menschlicher Unterstützung verpflichtet. Mein Dank gilt in erster Linie meinem verehrten Lehrer, Herrn Professor Dr. Bruno Boesch, für sein stets förderliches Interesse und wohlwollenden Rat während langer Jahre. Aufmunternde Hilfe durfte ich auch von den Herren Professoren Dr. Friedrich Maurer und Dr. Werner Besch sowie von Frau Professor Dr. Xenia von Ertzdorff und Herrn Dozenten Dr. Gerhard Meissburger erfahren. Für freundliche Hinweise danke ich Frau Professor Dr. Johanne Autenrieth und den Herren Professoren Dr. Werner Fechter, Dr. Lutz Röhrich und Dr. Hugo Steger. Besonders danken möchte ich Herrn Dr. Konrad Kunze für zahlreiche Anregungen wie für die mühevolle Durchsicht des Manuskripts. Nicht zuletzt danke ich herzlich meiner Frau, die durch ihre unermüdliche Mitsorge, Rücksicht und Ermunterung das Entstehen dieses Werkes ermöglicht hat. Ihr sei das Buch gewidmet.

$\mathrm{Zu}$ danken habe ich der J. B. Metzlerschen Verlagsbuchhandlung für die Aufnahme der Arbeit in die Reihe ,Germanistische Abhandlungen<, sowie der Deutschen Forschungsgemeinschaft für eine Druckkostenbeihilfe. 


\section{INHALT}

EINLEITUNG: Forschungslage und Fragestellung . . . . . . . . . . . . . . . . 1

I. Der Begriff Allegorie. . . . . . . . . . . . . . . . . . . . . . . . . 7

1. Der formale Aspekt der Allegorie . . . . . . . . . . . . . . . . . . . . . 7

1.1 Die Stellung der Allegorie in der Rhetorik . . . . . . . . . . . . . . . . 7

Griechen 8, Cicero 8, Herennius-Rhetorik 8, Quintilian 9, Schulgrammatiker 11, Matthaeus von Vendôme 13, Galfred von Vinsauf 13, Eberhard der Deutsche 14

1.2 Das theologische Verständnis der Allegorie . . . . . . . . . . . . . . . 15

Juden 15, Origenes 16, Mittelalter 17, Kritik an der Allegorese 19, Unterscheidung res - verba 21, Zusammenfassung 21

2. Bestimmung des Allegorie-Begriffs . . . . . . . . . . . . . . . . . 22

2.1 historisch . . . . . . . . . . . . . . . . . 22

a) im Mittelalter . . . . . . . . . . . . . . . . . . . . 22

Vermischung beider Stränge 23, allegorische Auslegung bzw. Neuschöpfung 25, dichterische significatio-Lehre 26, Zusammenfassung 26

b) Moderne Auffassungen und ihre Absetzung gegen den mittelalterlichen

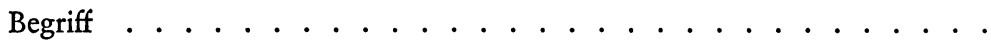

Ablehnung der Allegorie 28, Voraussetzungen der Begriffsbestimmung 29, neuzeitliche Bestimmungen 30, Allegorie - Symbol 30, mittelalterliche Sicht 34, Begriffsbestimmung 35

2.2 systematisch: Abgrenzung gegen verwandte Begriffe . . . . . . . . . . . 36

Typologie 36, Allegoristik 40, Metapher 41, Rätsel 42, Parabel 43, bîspel 44; Zusammenfassung 44

Il. Die mittelhochdeutsche Minneallegorie . . . . . . . . . . . . . . . 45

A. Die Gestaltung der Minneallegorie . . . . . . . . . . . 45

1. Minnelehre - Minneallegorie . . . . . . . . . . . . . . . . 45

1.1 ,Minnerede 6 . . . . . . . . . . . . . . . . . . . . . . . . 46

a) Begriff . . . . . . . . . . . . . . . . . . . . . . . . . . 46

b) Uberblick über die Minnereden . . . . . . . . . . . . . . . . . . . 47

c) Tradition der deutschsprachigen Minnelehre . . . . . . . . . . . . . . 52

1.2 ,Personifikationsdichtung ${ }^{6}$. . . . . . . . . . . . . . . . . . . 54

a) Gruppierung . . . . . . . . . . . . . . . . . . . . . . . . . . . . 55

b) Gedichte mit isolierter Personifikation . . . . . . . . . . . . . . . . 61 
c) Gedichte mit mehreren Personifikationen . . . . . . . . . . . . . . 63

d) Konrads von Würzburg „Klage der Kunst" . . . . . . . . . . . . . . 68

e) Personifikation und Allegorie . . . . . . . . . . . . . . . . . . . . 73

f) allegorische Personifikationsdichtungen . . . . . . . . . . . . . . 78

Gerichtsdichtungen 79, sonstige Motive 87

g) Typus der Personifikationsallegorie . . . . . . . . . . . . . . . . . . 89

1.3 ,Konstruktionsallegorie ${ }^{6}$. . . . . . . . . . . . . . . . . . . . . 91

a) eingebaute Allegorien . . . . . . . . . . . . . . . . 91

b) selbständige Allegorien . . . . . . . . . . . . . . . . . . . . 95

c) mehrere Allegorien nebeneinander . . . . . . . . . . . . . . . . . . 98

Konstruktionsallegorie und Personifikationen 99

d) Typus der Konstruktionsallegorie . . . . . . . . . . . . . . . . . . 101

1.4 Gegenüberstellung der allegorischen Typen . . . . . . . . . . . . . 101

2. Aussage der Minneallegorien . . . . . . . . . . . . . . . . . 104

2.1 Wesen der Minne . . . . . . . . . . . . . . . . . 104

a) Entstehung der Minne . . . . . . . . . . . . . . . . . 105 spekulativ 105 , religiös 106 , „von natur“ 107

b) Personifikation und Wesensbestimmung der Minne . . . . . . . . . 110 antike Terminologie und Attribute 110, mittelalterl. Terminologie: Minne/ Venus 112, Minne/Liebe 114

2.2 Wirkung der Minne . . . . . . . . . . . . . . . . . 117

2.3 Gesellschaftliche Wertung der Minne . . . . . . . . . . . . . . . . 118

a) Bedeutung der zentralen Sechsergruppierung der Tugenden . . . . . . . 119

Staete 120, Triuwe 122, Zucht 124, Scham 125, Liebe 126, Liebe/Minne 127, Ere 128

b) Aussage der Personifikationsdichtungen . . . . . . . . . . . . . . . . 130

2.4 Aussage der verschiedenen allegorischen Formen . . . . . . . . . . . 137

3. Räume und Handlungsmodelle der Minneallegorie . . . . . . . . . . . . . . 138

3.1 Einleitungstopoi . . . . . . . . . . . . . . . . . . . . . . . . 139

a) Traum . . . . . . . . . . . . . . . . . . . . . . . 139

b) Entführung . . . . . . . . . . . . . . . . . . . . . . . . . 142

c) Aventiureneingang . . . . . . . . . . . . . . . . . . . . . . . . . 143

d) Natureingang . . . . . . . . . . . . . . . . . . . . . . . . 145

e) Spaziergang . . . . . . . . . . . . . . . . . . . . . . . . . . . . . . . . . . . 146

3.2 Der Topos des zentralen Handlungsortes . . . . . . . . . . . 150

3.3 Architektur . . . . . . . . . . . . . . . . . . . 156

a) Haus/Stadt . . . . . . . . . . . . . . . . . . . . . . 156

b) Kloster . . . . . . . . . . . . . . . . . . . . . . . . . . . . . 162

c) Burg/Bergpalast . . . . . . . . . . . . . . . . . . . . . . . . . . 172

3.4 Auseinandersetzungen . . . . . . . . . . . . . . . . . . . . . . . 180

a) Gericht ． . . . . . . . . . . . . . . . . . . . . . . . . . . . . 181

b) Kampf . . . . . . . . . . . . . . . . . . . . . . . . . . . . . . 183 


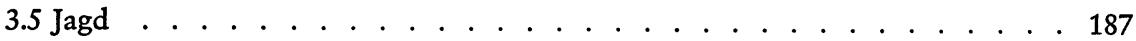

3.6 Zusammenfassung . . . . . . . . . . . . . . . . . . . . 194

4. Form der Minneallegorie. . . . . . . . . . . . . . . . . . . 196

4.1 Durchführung der Allegorie . . . . . . . . . . . . . . . . . 196

4.2 Formelemente . . . . . . . . . . . . . . . . . . 201

a) Metrik . . . . . . . . . . . . . . . . . . . . . . 201

b) Stil . . . . . . . . . . . . . . . . . . . . . . . 202

c) didaktische Elemente . . . . . . . . . . . . . . . . . 206

d) lyrische Elemente . . . . . . . . . . . . . . . . . . . . . 207

e) epische Elemente . . . . . . . . . . . . . . . . . 209

5. Die ,Gattung der Minneallegorie - Gestaltungsprinzipien . . . . . . . . 210

5.1 Frage nach der Einheit der ,Gattung' . . . . . . . . . . . . . 210

a) formale Einheit . . . . . . . . . . . . . . . . . 211

b) Aussage-Einheit . . . . . . . . . . . . . . . . . . . . . . . . . . . . . 214

c) Gestaltungseinheit . . . . . . . . . . . . . . . . . . . . 216 zwei Analysen: „Minneburg“ 216, „Der neuen Liebe Buch“ 223; Ergebnisse 229

5.2 Gattungszugehörigkeit der Minneallegorie . . . . . . . . . . . 230

5.3 Kriterien der Wertung . . . . . . . . . . . . . 231

B. Die Funktion der Minneallegorie . . . . . . . . 232

1. Erkenntniskritische Funktion . . . . . . . . . . . . 233

1.1 Nominalismus und Minneallegorie . . . . . . . . . . . . . . . 233

Verhältnis des Einzelnen zum Allgemeinen in der Minneallegorie 233, drei Funktionstypen 234, Folgerungen 235; Erfahrung und dichterisches Ich 237

1.2 Tendenz der Allegorie zur Ganzheit . . . . . . . . . . . . . 239

1.3 Anspruch auf Wahrheitserkenntnis . . . . . . . . . . . . 240

2. Moralische Funktion . . . . . . . . . . . . . . 241

3. Asthetische Funktion . . . . . . . . . . . . . . . . . 242

3.1 Beurteilung als Spiel . . . . . . . . . . . . . . 242

3.2 Erhebung des Inhalts . . . . . . . . . . . . . . . . . 244

3.3 Behandlung des Bildes . . . . . . . . . . . . . . 245

in den drei Allegorietypen 245, Trennung in zwei Richtungen 246, Dualismus der Allegorie 247

4. Vereinigung der theologischen und ästhetischen Funktion . . . . . . . . . 249

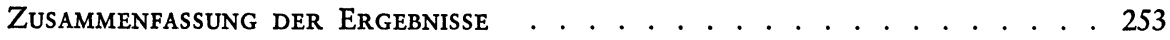

Literatur . . . . . . . . . . . . . . . . 255

Register . . . . . . . . . . . . . . . . 268 


\section{ABKÜRZUNGEN}

AfdA

ATB

BLV

DTM

DU

DVjs

DWb

ELH

Euph

GRM

Kraus, LD

LS

LThK

MR

PBB

PL

RAC

RDK

RGG

RL

ThWbzNT

VL

ZfdA

$\mathrm{ZfdPh}$
Anzeiger für deutsches Altertum und deutsche Literatur.

Altdeutsche Textbibliothek.

Bibliothek des literarischen Vereins in Stuttgart.

Deutsche Texte des Mittelalters.

Der Deutschunterricht. Beiträge zu seiner Praxis und wissenschaftlichen Grundlegung.

Deutsche Vierteljahrsschrift für Literaturwissenschaft und Geistesgeschichte.

Deutsches Wörterbuch. Hg. von J. und W. Grimm, 32 Bde., Leipzig 1854-1956.

A Journal of English Literary and History.

Euphorion. Zeitschrift für Literaturgeschichte.

Germanisch-romanische Monatsschrift.

Liederdichter des 13. Jahrhunderts, hg. von C. v. Kraus (s. Quellen II). Lassbergs Lieder Saal (s. Quellen I).

Lexikon für Theologie und Kirche. 2. Aufl. hg. von J. Höfer und K. Rahner, 10 Bde., Freiburg 1957-1965.

Mittelhochdeutsche Minnereden. Bd. I und II (s. Quellen I).

Beiträge zur Geschichte der deutschen Sprache und Literatur.

Patrologia latina, hg. von Migne (s. Quellen III).

Reallexikon für Antike und Christentum. Sachwörterbuch zur Auseinandersetzung des Christentums mit der antiken Welt. Hg. von Th. Klauser, bisher 6 Bde., Stuttgart $1950 \mathrm{ff}$.

Reallexikon zur deutschen Kunstgeschichte. Begr. von O. Schmitt, hg. von L. H. Heydenreich und K. A. Wirth, bisher 4 Bde., Stuttgart $1937 \mathrm{ff}$.

Die Religion in Geschichte und Gegenwart. Handwörterbuch für Theologie und Religionswissenschaft. 3. Aufl. hg. von K. Galling, 6 Bde., Tübingen 1957-1962.

Reallexikon der deutschen Literaturgeschichte. 2. Aufl. hg. von W. Kohlschmidt und W. Mohr, bisher 2 Bde., Berlin $1958 \mathrm{ff}$.

Theologisches Wörterbuch zum Neuen Testament. Begr. von G. Kittel, hg. von G. Friedrich, bisher 7 Bde., Stuttgart $1932 \mathrm{ff}$.

Die deutsche Literatur des Mittelalters. Verfasserlexikon. Hg. von W. Stammler und K. Langosch, 5 Bde., Berlin/Leipzig 1933-1955.

Zeitschrift für deutsches Altertum und deutsche Literatur.

Zeitschrift für deutsche Philologie. 